\title{
Spectroscopic measurement of cortical nitric oxide release induced by ascending activation
}

\author{
N. Espinosa, J. Cudeiro, J. Mariño
}

\begin{abstract}
The transition from sleep to the awake state is regulated by the activation of subcortical nuclei of the brainstem (BS) and basal forebrain $(\mathrm{BF})$, releasing acetylcholine and glutamate throughout the cortex and inducing a tonic state of neural activity. It has been suggested that such activation is also mediated by the massive and diffuse cortical release of nitric oxide (NO). In this work we have combined the spectroscopic measurement of NO levels in the somatosensory cortex of the cat through its marker methemoglobin, as well as two other hemodynamic markers (oxyhemoglobin - oxy $\mathrm{Hb}$ - and deoxyhemoglobin - deoxyHb), together with the electrical stimulation of BS and BF - to induce an experimental transition from a sleep-like state to an awake-like mode. The results show an increase of NO levels either after BS or BF activation. The response induced by BS stimulation was biphasic in the three studied markers, and lasted for up to $30 \mathrm{~s}$. The changes induced by BF were monophasic lasting for up to $20 \mathrm{~s}$. The systemic blockade of NO production abolished the observed responses to BS whereas responses to BF stimulation were much less affected. These results indicate a crucial role for NO in the neuronal activation induced by the ascending systems.
\end{abstract}

Key words. hemodynamics; sleep; somatosensory cortex; spectroscopy

\section{Introduction}

Arousal is the consequence of an orchestrated change in cortical and subcortical structures. The transition from sleep to the awake state is mainly induced by brainstem (BS) and basal forebrain (BF) neurons (Steriade et al., 1993a), which are part of the classic activating ascending system. As a result of such activation, the cortical release of acetylcholine $(\mathrm{ACh})$ and glutamate $(\mathrm{Glu})$ disrupts the sleep slow oscillatory activity and promotes a transition to the characteristic tonic state of the awake brain (for a review see Steriade et al., 1997). It has been suggested that nitric oxide (NO) is also an important actor in this activation, mainly through its release by cholinergic BF neurons sending axons to the cortex (Bickford et al., 1994 and Cudeiro et al., 2000) and by cortical nitrergic neurons (Cudeiro et al., 1997).

Brain sleep mechanisms have been extensively studied using anesthetized cats as a model to reproduce the slow-wave sleep and its dynamics (Steriade et al., 1991a, Steriade et al., 1991b and McCormick and Bal, 1997). Also, under experimental conditions, the global cortical activity can be efficiently modified through microstimulation of either the peribrachial region (PBr; Moruzzi and Magoun, 1949, Francesconi et al., 1988, Hartveit et al., 1993, Uhlrich et al., 1995 and Wolfe and Palmer, 1998), located in the BS, or the nucleus basalis of Meynert (NB; Metherate et al., 1992 and Mariño and Cudeiro, 2003), located in the BF. The BS contains cholinergic, nitrergic and catecholaminergic neurons sending axons to BF and the thalamus (Steriade et al., 1988). BF neurons extensively release ACh throughout the cortex and, as indicated, probably NO, while thalamocortical neurons release Glu. In the anesthetized cat, the activation of such systems has been proved to be a useful tool to study the sleepwake mechanisms (Steriade et al., 1991b, Li et al., 1999 and Mariño and Cudeiro, 2003).

We have previously shown that the systemic and local blockade of NO synthesis (by 7-nitroindazole and L-NOArg respectively) reduces the tonic activation of cortical neurons after subcortical stimulation (Mariño and Cudeiro, 2003). Nonetheless, as far as we know, no direct measurements of that supposed massive NO release has been reported yet. Here, using intracortical spectroscopy in the somatosensory cortex (SSC), we have measured the in vivo dynamics of cortical NO levels during sleep-like slow oscillatory activity (induced by anesthesia) and after the activation induced by BS and BF regions, together with the measurements of two hemodynamic markers, oxyhemoglobin (oxyHb) and deoxyhemoglobin (deoxyHb). Our central hypothesis was that, during the intense activation induced by the ascending systems, there is a diffuse and acute production of neuronal NO in the cortex, thus collaborating in the transition from sleep to the wake state. 


\section{Experimental procedures}

\section{Experimental preparation}

Experiments were performed on 4 adult cats of either sex. Anesthesia was induced with ketamine $(15 \mathrm{mg} / \mathrm{kg}$ - Pfizer, Madrid, Spain) and xylazine (3 mg/kg - Sigma-Aldrich, Madrid, Spain) i.m. and maintained with isoflurane (1.5-2\% for surgery, and $0.5-1 \%$ for maintenance, Abbot) in nitrous oxide (70\%) and oxygen (30\%). Animals were paralyzed with gallamine triethiodide (loading dose of $40 \mathrm{mg}$, maintenance $10 \mathrm{mg} / \mathrm{kg} / \mathrm{h}$ i.v., SIGMA) and artificially ventilated; the end tidal $\mathrm{CO}_{2}$ concentration was adjusted to $3.8-4.2 \%$, body temperature was maintained at $37-38{ }^{\circ} \mathrm{C}$, and heart rate was continuously monitored. Animals were suspended on a stereotaxic frame and the pressure points were infiltrated with lidocaine. The level of isoflurane was chosen to achieve a state of deep anesthesia and particular care was taken to keep a stable pattern of delta $(1-4 \mathrm{~Hz})$ slow oscillatory activity throughout the entire experiment. Three craniotomies were performed to insert optical fibers and bipolar tungsten electrodes (details below). All exposed cortical surfaces were bathed in saline to prevent desiccation. At the end of experiments animals were killed by an anesthetic overdose. All animal work was conducted according to national and international guidelines (Spanish Physiology Society and the European Communities Council Directive of November 24, 1986 (86/609/EEC)). The study was approved by the University of A Coruña Ethics Committee (CE-UDC30/1/09).

\section{Electrophysiological and spectroscopy recordings}

Electrical and spectroscopic measurements were obtained simultaneously in the primary SSC (S1; coordinates: anterior, 21-23; lateral, 8-10) at a depth of 1-1.5 mm. Electrocorticogram (ECoG) in S1 was continuously recorded and stored (Plexon Inc, Dallas, TX, USA) through a bipolar and concentric electrode at a sampling rate of $2 \mathrm{kHz}$ and filtered on-line with a band-pass of $0.1-500 \mathrm{~Hz}$ analog filter (AM Systems, model 1700).

The method to monitor NO lays in the fact that NO reacts with oxyHb to form methemoglobin (metHb). Thus, metHb levels are proportional to the concentration of NO and can be used as a surrogate marker of such substance (Feelisch et al., 1996, Kelm et al., 1997, González-Mora et al., 2002 and Rivadulla et al., 2011). According to this, we used spectroscopy to record the levels of metHb as well as oxy- and deoxyHb. Spectroscopic signals were obtained using two optical fibers (model FCBUV 100-3-2SMA; diameter $100 \mu \mathrm{m}$ ) arranged in parallel and attached to the electrode. Distance from the electrode tip to the fibers end (located slightly below, see Fig. 2E) was between 100 and $200 \mu \mathrm{m}$. Light in the range of $600-700 \mathrm{~nm}$ from a halogen lamp was passed through one of the optical fibers and the scattered light was collected by the other. Output was directed to a linear CCD detector (Ocean Optics, Eerbeek, Netherlands) via a compact built-in monochromator and sampled at a rate of $100 \mathrm{~Hz}$ with steps of $0.35 \mathrm{~nm}$ in the wavelength domain. 


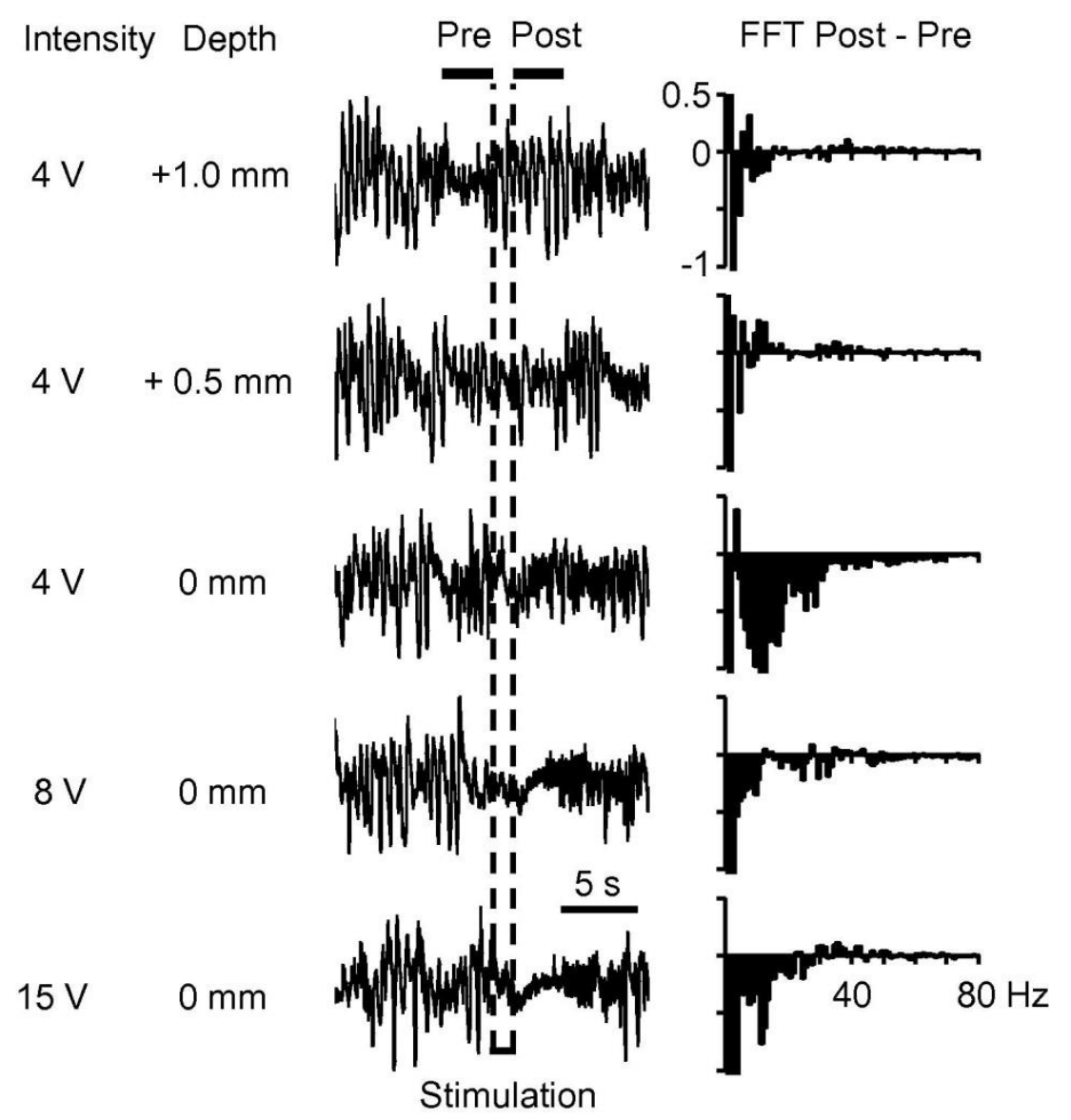

Fig. 1. Electrical stimulation effect obtained for different stimulation intensities and electrode distances from the stereotaxic position $(0 \mathrm{~mm})$ of the parabrachial region of BS. Both ECoG wave (left) and its power spectral density (right) reveal changes in low-and high-frequency components. The analysis included the $5 \mathrm{~s}$ prior stimulation (Pre), and the $5 \mathrm{~s}$ after stimulation (Post). See "Experimental procedure" section for details. 
A
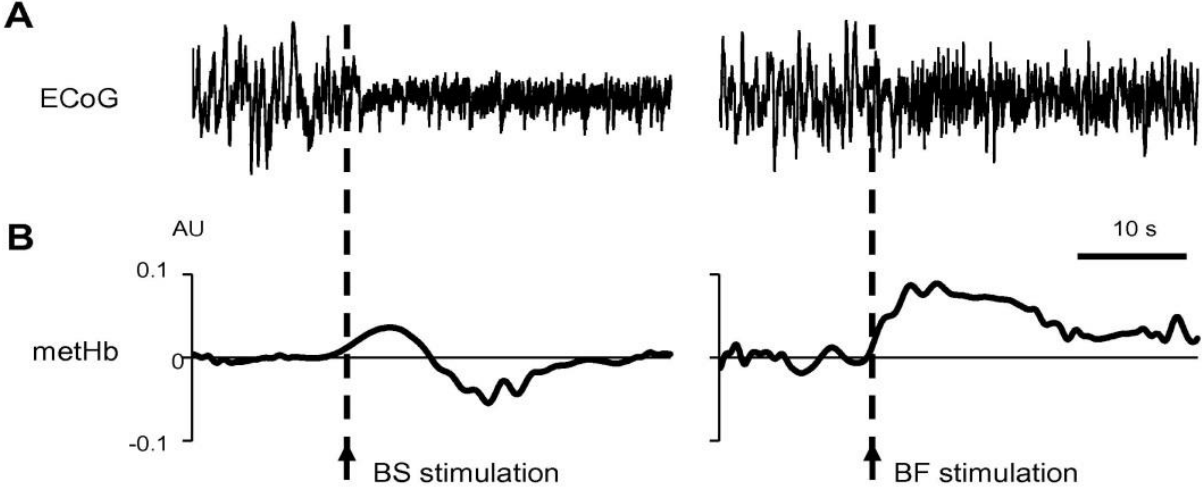

C
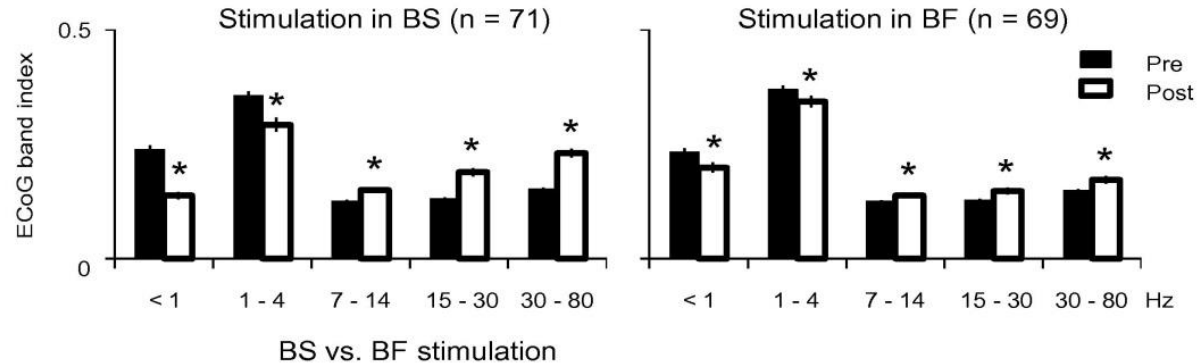

D

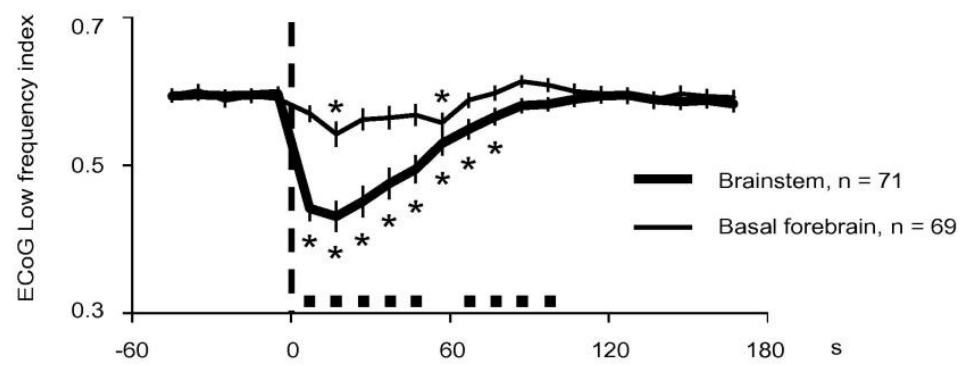

E

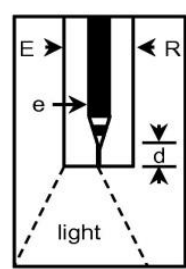

Fig. 2. (A) ECoG activity recorded in S1 before and after electrical stimulation of BS and BF in one cat. (B) Cortical metHb dynamics simultaneously recorded. $Y$ axis is the relative concentration of metHb obtained by solving Eq. (4) and expressed in arbitrary units (AU). (C) $\mathrm{ECoG}_{\text {index }}$ for frequency bands of interest before (solid bars) and after (open bars) electrical stimulation in BS and BF. Recordings were performed in four cats. (D) Low-frequency index analysis. For each brain structure studied, values (mean and SEM) at Post were contrasted with the values Pre stimulation. Dashed line indicates stimulation. Each time-point represents bins of $10 \mathrm{~s}$. Asterisks indicate significant effects at different time points compared to the Pre values. Black squares represent significant differences in the response observed in the two structures at different time-points after the stimulation. E) Schematic drawing of the bipolar tungsten electrode (e) and optical fibers. Emitter (E) and the receiver (R) fibers deliver and collect the light respectively. Distance from the electrode tip to the fibers end (d) was set to 100-200 $\mu \mathrm{m}$.

To convert the sample intensity recordings $\mathrm{S}$ to absorbance values the protocol was as follows: with the fibers located in the tissue, the emitted light was turned off and the dark intensity D was recorded. Next, light was turned on and the reference intensity $\mathrm{R}$ was recorded. Then, for a sample intensity at wavelength $\lambda$ and recorded at time $t, \mathrm{~s} \lambda(\mathrm{t})$, the corresponding absorbance, ${ }_{\mathrm{A} \lambda}(\mathrm{t})$, was calculated using the following equation:

$$
A(t)_{\lambda}=-\log _{10}\left(\frac{S(t)_{\lambda}-D_{\lambda}}{R_{\lambda}-D_{\lambda}}\right)
$$

where $D \lambda$ and $R \lambda$ are the dark and reference intensities for a wavelength $\lambda$ respectively. Because absorbance is a dimensionless parameter, the value is expressed in arbitrary units (AU). 


\section{Subcortical stimulation and cortical activity modulation}

The global cortical activity was efficiently modulated - as indicated by the ECoG recordings - by the combination of anesthesia and electrical stimulation. The continuous anesthetic administration maintained a slow oscillatory pattern within the delta range (Fig. 1, left part of the ECoGs). The temporal transition to an awake-like pattern was achieved through the application of electrical stimulation either in BS or BF (sequentially and randomly through electrical microstimulation at intervals of 2-8 min). Trains of rectangular cathodal shocks $(0.05 \mathrm{~ms}, 0.1-1 \mathrm{~mA})$ were delivered at a frequency of $50 \mathrm{~Hz}$ for a period of $2 \mathrm{~s}$ through bipolar electrodes (insulated except for $500 \mu \mathrm{m}$ at the tip; contacts separated by $500 \mu \mathrm{m}$ ). Electrodes were positioned and held in place following a procedure published elsewhere (Mariño and Cudeiro 2003). It allowed us to precisely localize the subcortical loci for electrical stimulation rendering histological reconstruction, in this case, unnecessary. The procedure was as follows. Horsley-Clarke coordinates were used to achieve the $\mathrm{PBr}$ region in the $\mathrm{BS}$ (anterior, 0; lateral 4; ventral, -2 ) and the NB in the $\mathrm{BF}$ (anterior, 10-13; lateral, 8.5-10; ventral, -1 to 0 ). For each electrode, the final position in the vertical axes was adjusted monitoring the stimulation effect on the ECoG activity. Fig. 1 depicts one example of the successive ECoG recordings and frequency spectra obtained during the descent of one stimulating electrode toward the $\mathrm{PBr}$ region. A low-intensity (typically $4 \mathrm{~V}$ ) 2-s train was delivered to localize the optimal position inside each brain region. The search procedure started with the electrode located $4 \mathrm{~mm}$ above the theoretical stereotaxic coordinates and the location was tested in steps of $0.25 \mathrm{~mm}$. Once a clear effect on the ECoG was evoked, the voltage was adjusted (between 4 and $15 \mathrm{~V}$ ) to the minimum intensity capable of optimally inducing a low-amplitude and high-frequency activity in S1 (see waveforms and fast Fourier transform (FFT) analysis in Fig. 1). The same procedure was applied to locate the appropriate stimulating location in the BF. Such effective modulation of the ECoG was indicative of a suitable stimulation of the ascendant pathways (Mariño and Cudeiro 2003).

\subsection{Pharmacology}

In order to inhibit the enzyme neuronal NOS (neuronal nitric oxide synthase), responsible of neuronal NO production, 7-nitroindazole (7NI; SIGMA) was administered i.v. over $15 \mathrm{~s}(3.5 \mathrm{mg} / \mathrm{kg}$ dissolved in $0.1 \mathrm{ml}$ DMSO (SIGMA); de Labra et al., 2009).

\subsection{Data analysis}

The FFT was used to obtain the power spectral density from ECoG recordings for periods of 5-10 s before and after electrical stimulation. Frequency ranges of interest were adjusted to previously described cat sleep rhythms (for a short review, see Steriade et al. (1993b)) according to the following classification: <1 Hz (slow), 1-4 Hz (delta), 7-14 Hz (spindle), 15-30 Hz (beta) and 30-80 Hz (gamma). To evaluate the electrical stimulation effect on each frequency band, an ECoG index was defined as follows:

$$
\mathrm{ECoG}_{\text {index }}\left(f_{n}\right)=\frac{\sum \operatorname{PSD}\left(f_{n}\right)}{\sum \operatorname{PSD}\left(f_{T}\right)}
$$

where $\sum \operatorname{PSD}(f)$ is the cumulative sum of the power spectral density for the frequency range $f . f n$ is the frequency range for one of the rhythms aforementioned and $f T$ is the frequency range between 0 and $80 \mathrm{~Hz}$ (DC component filtered previously). Specifically, to evaluate the power of the lowest frequency waves in the ECoG, a low-frequency index (LFi) was defined as follows:

$$
\mathrm{LFi}=\frac{\sum \operatorname{PSD}\left(f_{L}\right)}{\sum \operatorname{PSD}\left(f_{T}\right)}
$$

where $f L$ is the frequency range below $4 \mathrm{~Hz}$. 
For the spectroscopic recordings, absorbance values were filtered (Savitzky-Golay filter) and linearly interpolated in the wavelength domain (range $600-700 \mathrm{~nm}$, steps of $1 \mathrm{~nm}$ ) and in time domain to normalize timestamps $(100 \mathrm{~Hz}, t=0 \mathrm{~s}$ for the electrical stimulation onset). The relative concentration of oxyHb, deoxyHb and metHb were calculated by means of the Lambert-Beer's law solving the following Eq. (4).

$$
\left[\begin{array}{c}
\Delta \text { oxyHb }(\mathrm{t}) \\
\Delta \text { deoxyHb }(\mathrm{t}) \\
\Delta \text { metHb }(\mathrm{t})
\end{array}\right]=\left[\begin{array}{c}
\alpha_{\text {oxyHb }}^{\lambda_{1}} \alpha_{\text {oxyHb }}^{\lambda_{2}} \alpha_{\text {oxy }}^{\lambda_{3}} \\
\alpha_{\text {deoxyHb }}^{\lambda_{1}} \alpha_{\text {deoxyHb }}^{\lambda_{2}} \alpha_{\text {deoxyHb }}^{\lambda_{3}} \\
\alpha_{\text {metHb }}^{\lambda_{1}} \alpha_{\text {metHb }}^{\lambda_{2}} \alpha_{\text {metHb }}^{\lambda_{3}}
\end{array}\right] \cdot\left[\begin{array}{c}
\mathrm{A}_{\lambda_{1}}(\mathrm{t}) \\
\mathrm{A}_{\lambda_{2}}(\mathrm{t}) \\
\mathrm{A}_{\lambda_{3}}(\mathrm{t})
\end{array}\right]
$$

where $\alpha_{\text {oxyHb }}^{\lambda_{i}} \alpha_{\text {deoxyHb }}^{\lambda_{i}}$ Unknown node type: italicUnknown node type: italicioxyHbUnknown node type: italicUnknown node type: italicideoxyHb and $\boldsymbol{\alpha}_{\text {metHb }}^{\lambda_{i}}$ Unknown node type: italicUnknown node type: italicimetHb are the extinction spectra, at wavelength $\lambda$ iUnknown node type: italici, for oxy-, deoxy-, and metHb, respectively. Since A $\lambda \mathrm{i}(\mathrm{t}) \mathrm{AUnknown}$ node type: italici $(\mathrm{t})$ is the recorded absorbance value at wavelength $\lambda$ iUnknown node type: italici (see Eq. (1)), we solved Eq. (4) for different wavelength trios selected randomly within the range $600-700 \mathrm{~nm}$ (steps of $1 \mathrm{~nm}$; smaller steps did not modified the statistical significance of final results) until the whole wavelength range was covered. The extinction spectra reported by Zijlstra et al. (2000) was used for the corresponding wavelengths. As a result, for each relative concentration we obtained a distribution and the average value was taken as the estimated value at time $=t$. Finally, a wavelet filter was applied to oxy-, deoxy-, and metHb in the time domain using a family of Meyer (discrete approximation) wavelets.

Statistical analysis was done using paired $t$-test or Wilcoxon test. The hypothesis of normality was checked using the Kolmogorov-Smirnov test. Results were deemed to be significant when $p<0.05$. Both analytical and statistical analyses were performed using in-house software written in MATLAB (MathWorks, 2010).

\section{Results}

\section{Effects on ECoG activity of the electrical stimulation of activating ascendant pathways}

The sleep-like ECoG activity induced by anesthesia was modified by the electrical stimulation of BS and BF (Fig. 2A). The ECoG changed from a high-amplitude low-frequency pattern of activity to a lowamplitude high-frequency mode.

Electrical stimulation of BS $(n=71)$ and BF $(n=69)$ modified the sleep-like oscillatory activity induced by anesthesia in different ways. To quantify this phenomenon, the ECoG power spectral density was calculated $5 \mathrm{~s}$ before the onset and $5 \mathrm{~s}$ immediately after the offset of the electrical stimulation. The $\mathrm{ECoG}_{\text {index }}$ in the frequency of interest bands were calculated (see "Experimental procedure" section). Population analysis of the effects induced by BS ( Fig. 2C, left) and BF ( Fig. 2C, right) stimulation revealed a decrease in the low-frequency activity (below $4 \mathrm{~Hz}$ ) and an increase of higher frequency components (above $4 \mathrm{~Hz}$ ).

To analyze the persistence and strength of the electrical stimulation effects, the LFi (see "Experimental procedure" section) was calculated using 10-s bins. Fig. 2D reveals a fast drop of the LFi induced by BS activation, lasting for up to $60 \mathrm{~s}$ after the electrical stimulation, whereas BF stimulation induced significant changes $10 \mathrm{~s}$ after the stimulation and lasting only $10 \mathrm{~s}$ (with an apparent late rebound). Besides, not only the duration but also the magnitude of the effects induced on the ECoG by the electrical stimulation on BS and BF was different (the statistically significant differences are indicated by black squares in Fig. 2D).

\section{Effects on NO and hemodynamic markers of the stimulation of ascending pathways}

Spectrometric recordings simultaneous to the above-mentioned ECoG, revealed that those changes were accompanied by a patent increase in cortical metHb (Fig. 2B). Population analysis of the time course of changes in met-, deoxy-, and oxyHb induced by BS and BF stimulation is show in Fig. 3. To define statistical variations, the mean values during control condition, i.e. before the electrical stimulation, were compared with each value after the stimulation (paired $t$-test). The resulting time intervals with significant changes are shown by marks below the curves. 


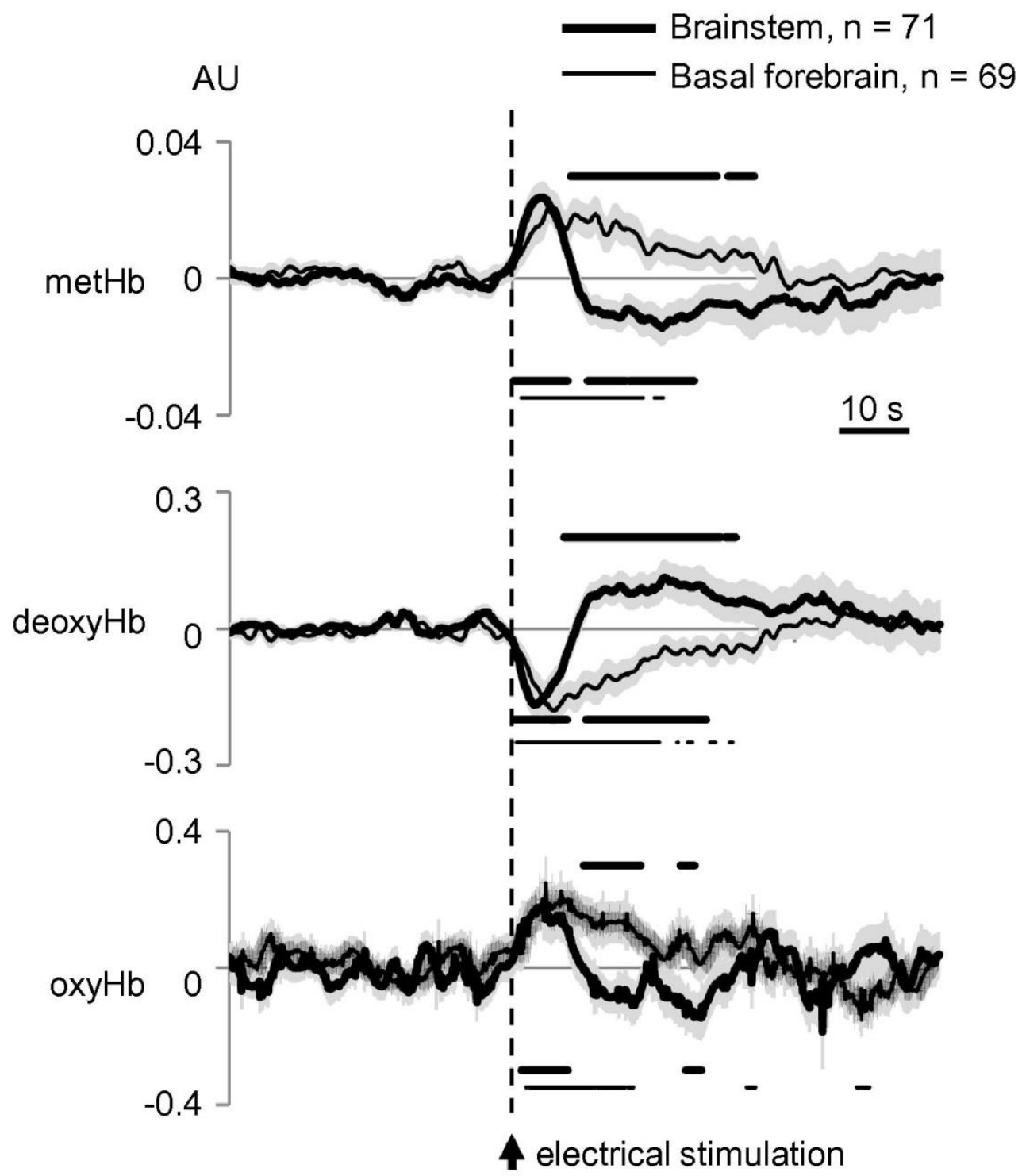

Fig. 3. BS and BF stimulation-induced responses at $\mathrm{S} 1$ on the three studied markers. Dots under curves show significant changes at different time-points after BS (thick dots) and BF (thin dots) stimulation compared to the average response $60 \mathrm{~s}$ before the stimulation. Dots above curves denote significant differences between temporal profiles for BS and BF stimulation. Shaded area represents SEM. Values obtained by solving Eq. (4) and expressed in arbitrary units (AU). Recordings were performed simultaneously with ECoGs analyzed in Fig. 2C, D (four cats).

Stimulation in BS (thick curves in Fig. 3) induced an increase of metHb, followed by a subsequent decrease, lasting in total for up to $30 \mathrm{~s}$ after the stimulation (as indicated by the reference lines below the curve). A similar biphasic change, but in the opposite direction, was observed for the deoxyHb. Changes in of oxyHb were less conclusive, showing an initial increase of its levels lasting for around $10 \mathrm{~s}$ after the stimulation, followed by a variable - although in some periods statistically significant - response.

With regard to BF stimulation (thin curves in Fig. 3), it induced, in contrast, a monophasic variation of the three markers. The mean response showed a clear increase of metHb levels, lasting for around $20 \mathrm{~s}$ after the stimulation; together with a decrease of deoxyHb and an increase of oxyHb of similar duration.

Finally, statistical comparison between the responses obtained with BS and BF stimulation revealed that both hemodynamic markers and metHb showed the same temporal pattern during the first $10 \mathrm{~s}$ after the end of the electrical stimulation. 


\section{Dissecting the role of $\mathrm{NO}$ on cortical activation}

As we had previously shown, the systemic application of 7NI reduced the efficacy of the ascendant activation to modify the ECoG slow pattern (Mariño and Cudeiro, 2003). In the present study we compared that phenomenon with the simultaneously recorded spectroscopic measurements.

The spectroscopic measurements allowed us to precisely follow the NO levels before and after 7NI injection. Fig. 4A shows the post-stimulus dynamics (average, $n=5$ ) of cortical metHb after BS and BF stimulation during control condition (top panel), peak drug effect (middle panel) and recovery from nNOS inhibition (bottom panel). To compare the metHb responses induced by stimulation, the resting value - mean value $40 \mathrm{~s}$ before electrical stimulation - was subtracted from each curve (see Fig. 4C). To quantify the effect of 7NI on NO as well as on the two hemodynamic markers, and having into account the specific time course of each response, the stimulation evoked responses were integrated for BS (10 s interval post stimulus) and BF (30 s interval post stimulus) ( Fig. 4B; intervals $\alpha$ depicted in Fig. 4A). After 7NI administration there was a clear impairment in the ability of the ascending activation to modify oxy-, deoxy- and metHb levels, mainly during 11-20 min after 7NI application. In addition, the electrical activity during this period contains a higher component on the low-frequency band ( Fig. 4B bottom panel). It is interesting to note that the resting values of the measured substances, but not the ECoG, were also affected by the nNOS blockade ( Fig. 4C). Similarly to the observed effects on the evoked responses, these changes lasted for up to $20 \mathrm{~min}$. 

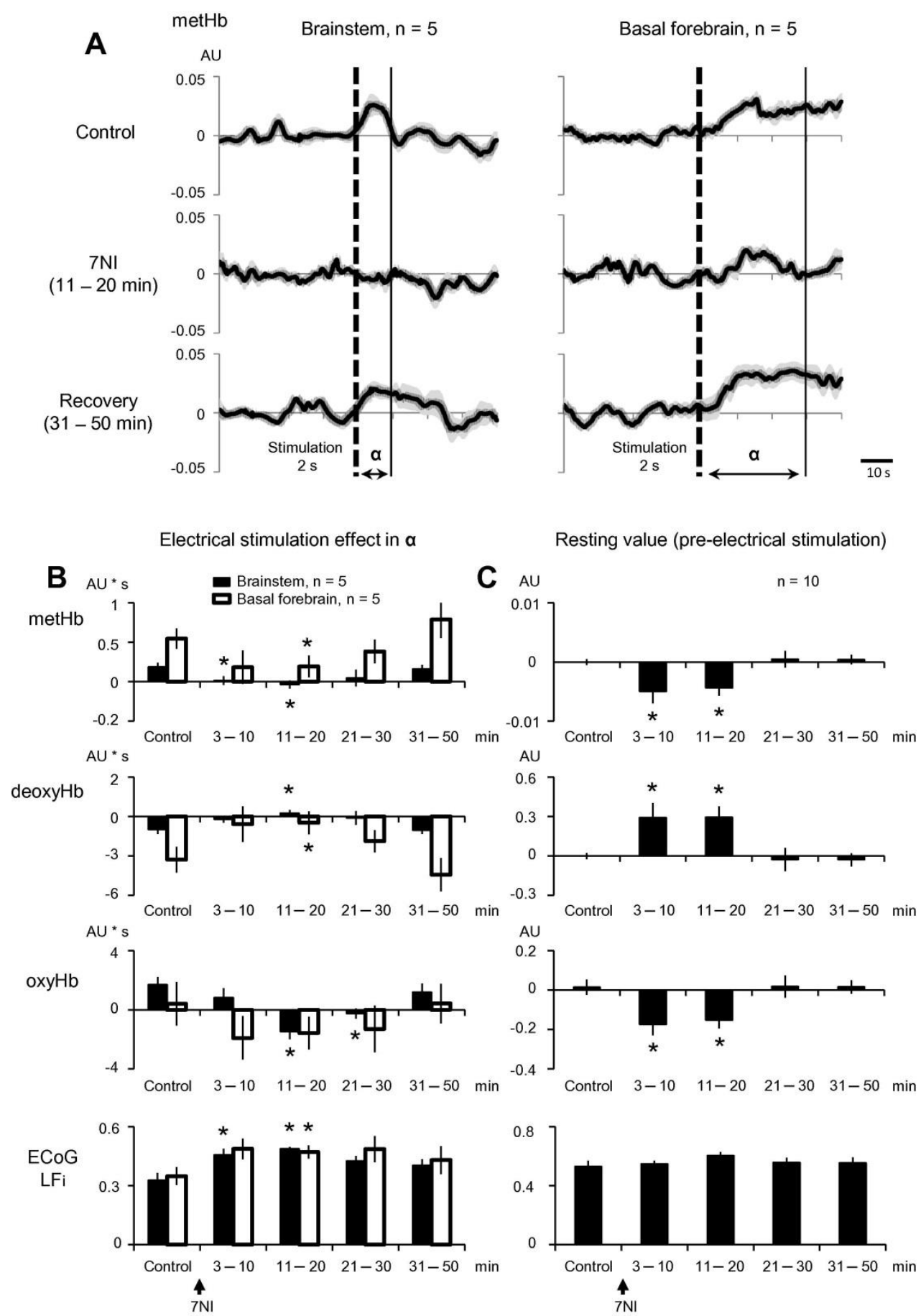

Fig. 4. (A) Effect on metHb before 7NI administration (Control; upper panel), during the peak drug effect (11-20 min after 7NI administration; middle panel) and during recovery (31-50 min; bottom panel) in response to BS (left panel) and BF (right panel) stimulation. Dashed line depicts the electrical stimulation $(2 \mathrm{~s})$ and $\alpha$ is the interval of interest. Shaded area denotes SEM for the five recordings obtained from three cats. (B) The area under the curve in $\alpha$ (A) for the three studied markers was compared during control and after 7NI injection for BS (solid bars) and BF (open bars) stimulation. Bottom panel shows the LFi calculated in $\alpha$. (C) Mean value of metHb and hemodynamic markers before stimulation calculated during control and at different intervals after 7NI injection. Values for BS and BF stimulation were pooled together $(n=10$ recordings). Bottom panel shows the LFi before stimulation. Asterisks indicate significant effects compared to control values. 


\section{Discussion}

The present study unveils the in vivo changes on NO dynamics in the cat cortex during the transition from a global pattern of synchronized slow activity to an awake-like mode. In previous works we suggested a direct role for the neuronal NO in the cortical activation induced by the ascending systems. We observed that the changes in ECoG activity induced by BF stimulation were practically abolished when administering 7NI i.p. ( Mariño and Cudeiro, 2003). Here, by means of spectroscopic continuous recordings, we have studied in detail the time course of NO release, and compared it with the responses induced by BF and BS stimulation.

Our results show that, in parallel to the categorical disruption of the slow ECoG activity evoked by BS and $\mathrm{BF}$ stimulation, there is an increase of NO levels immediately after the activation, lasting for $10 \mathrm{~s}$ (BS) or $20 \mathrm{~s}(\mathrm{BF})$. This fact strongly supports our hypothesis that the sudden and global release of NO throughout the cortex (we have measured a similar NO dynamics in primary visual cortex; not shown here) is directly contributing to the characteristic neuronal activation that occurs during the transition from slow to tonic activity. In the present work the spontaneous slow delta activity was induced by anesthesia, and cortical de-synchronization was transiently evoked by electrical stimulation; but the observed NO changes are likely to be of similar nature under natural sleep-wake transitions. The magnitude of the referred changes was smaller in metHb than in the other markers (note arbitrary unit axes in Fig. 3). It must be noted that BF stimulation evoked significant changes of shorter duration, an effect than can explain the smaller changes observed in the ECoG after this activation. An interesting possibility to complement our data could be the use of imaging techniques (e.g. optical imaging). It might provide spatial information to corroborate the "diffuse" increase in NO over the cortex. Certainly it will deserve attention in future experiments together with multiple spectroscopic measurements on different cortical areas.

Is this NO-mediated cortical activation dependent on cGMP production? Our experimental approach does not allow us to directly address this issue and we can only speculate. In the cerebral cortex, the diversity of NO production sites suggests a complex role in cortical processing and perhaps more than a single mode of action of NO. For instance, it has been shown that NO may be involved in the NMDAmediated release of noradrenaline and Glu suggesting that NO can have both direct actions and actions on modulatory processes (Montague et al., 1991). On the other hand previous findings from our group (Cudeiro et al., 1997) obtained with iontophoretic application of 8-bromo-cGMP suggest a simple modulation of the cGMP secondary messenger system. Such an action on cGMP has been widely reported in the literature (for reviews see Garthwaite, 1991, Moncada et al., 1991, Snyder and Bredt, 1991, Schuman and Madison, 1994 and Zhang and Snyder, 1995).

In an in vivo preparation like the one we used here, it is difficult to clearly separate the NO effects of the vascular and the neuronal compartments. Besides, it has been reported that the exposure of nitrous oxide might interfere with NO metabolism ( Zelinski et al., 2009 and Ohgami et al., 2010) and with NO background levels. Therefore, we cannot completely exclude a putative interference of the anesthesia with the observed levels of NO, although we trust that our main findings remain, since electrical stimulation produced an increment of NO. In any case, our goal was to discern the participation of neuronal NO in the massive cortical activation induced by the ascendant pathways. The inability of the ascending stimulation to induce such ECoG and metHb changes after the blockage of nNOS clearly points to a direct contribution of the neuronal NO in the activating process.

\section{Possible functional significances of BF and BS activations}

Having in mind that the relationship between $\mathrm{NO}$, oxyHb, and deoxyHb is not simple (because the vasodilation induced by $\mathrm{NO}$ is also influencing the hemodynamics and the oxy-deoxyHb equilibrium), our work indicates differential actions of both structures (BF and BS) on cortical neurovascular coupling. The present results suggest that changes in cerebral blood flow induced by the basalocortical pathway would be intimately linked to a vasodilation induced by NO. In fact, the monophasic and sustained hemodynamic changes induced by BF stimulation correlate well with the measured NO dynamics, but not with the neuronal activity observed after stimulation. This dissociation between neuronal and hemodynamic activity, previously observed in other brain regions, has been attributed to the participation of inhibitory networks (Ackermann et al., 1984 and Buzsaki et al., 2007). The fact that the basalocortical pathway also includes GABAergic fibers supports this hypothesis, allowing a marked hemodynamic change with a less robust response on cortical electrical activity. Previous studies have shown that the BF plays a key role in vigilance states and cognitive functions (Semba, 2000, Jones, 2003 and Jones, 2004). Because during an attentional process the system must be able to detect and process unexpected signals, a 
blood flow increase without a dramatic basal neuronal activity disturbance provides sufficient glucose and oxygen for a proper energy metabolism of the neural network.

$\mathrm{PBr}$ stimulation, in contrast, involves not only a cortical cholinergic and nitrergic release through $\mathrm{BF}$ but also a glutamatergic effect induced via the thalamus that would be the origin of a stronger effect on ECoG as well as the post-stimulus over- and undershoots observed in deoxy- and metHb, respectively. The ability to interfere on cortical activity and the fact that it receives inputs from the lateral parts of the preoptic area (Swanson et al., 1987), an active sleep-promoting structure (Szymusiak and McGinty, 1986, Sherin et al., 1996 and Szymusiak et al., 2001), supports the hypothesis that the PBr plays a key role in the transitions of the sleep-wake cycle.

\section{Implications in the blood oxygenation level dependent (BOLD) signal interpretation}

BOLD signal is a parameter widely used to estimate the cerebral activity which has given rise to functional network maps on several species (Buckner et al., 2008, Pawela et al., 2008, Mantini et al., 2011 and White et al., 2011) in virtue of a supposed neurovascular coupling. BOLD response is based on the paramagnetic property of deoxyHb. Thus, changes of deoxyHb concentration in a vascularized area subjected to a magnetic field are reflected as a change in the magnetic resonance (MR) signal. Curiously, neural activity leads to an increase in MR signal even though deoxyHb decreases MR signal (Ogawa et al., 1990). Given the diamagnetic nature of oxyHb, and supported by optical imaging studies (Malonek and Grinvald, 1996), it is believed that this effect occurs because oxyHb displaces deoxyHb that had been suppressing the MR signal intensity. Our data provide a concomitant cause based on the paramagnetic property of metHb being even stronger than deoxyHb. It is due to the fact that unpaired electrons in heme iron of hemoglobin provide paramagnetic properties. Thus, deoxyHb has four unpaired electron and metHb has five (Nitz et al., 2010). As a result, when NO release is associated to a neural activation, a portion of the increase in MR signal might be due to the influence of metHb increase as well. In fact, previous results show an impact of metHb in BOLD response (Duewell et al., 1996 and Leung and Moody, 2010, but see Pang et al., 2010) and a recent study has encouraged exploring their clinical implications (Aamand et al., 2013). Finally, we do not discard that other techniques susceptible to high concentrations of metHb, as MR spectroscopy (Gujar et al., 2005), could be affected by NO production.

\section{Conclusions}

The electrical stimulation of the activating ascending systems induces profound changes on cortical (electrical and vascular) activity. We have outlined the role of the neuromodulator NO in this process. Our results reveal a transient cortical increase of the NO marker metHb after the induction of a global brain activation, a change that is abolished after the inhibition of NO synthesis. The present study supports the hypothesis that NO collaborates in the transition from slow-wave sleep to tonic activity in the cortex.

\section{Funding}

This work was supported by Xunta de Galicia (INCITE09 137272 PR and Ayudas a Grupos Consolidados).

\section{References}

Aamand et al., 2013. R. Aamand, T. Dalsgaard, Y.C. Ho, A. Møller, A. Roepstorff, T.E. Lund. A NO way to BOLD?: Dietary nitrate alters the hemodynamic response to visual stimulation. Neuroimage, 83 (2013), pp. 397-407

Ackermann et al., 1984. R.F. Ackermann, D.M. Finch, T.L. Babb, J. Engel Jr. Increased glucose metabolism during long-duration recurrent inhibition of hippocampal pyramidal cells. J Neurosci, 4 (1984), pp. 251-264

Bickford et al., 1994. M.E. Bickford, A.E. Günlük, S.C. Van Horn, S.M. Sherman. GABAergic projection from the basal forebrain to the visual sector of the thalamic reticular nucleus in the cat. J Comp Neurol, 348 (1994), pp. 481-510

Buckner et al., 2008. R.L. Buckner, J.R. Andrews-Hanna, D.L. Schacter. The brain's default network. Ann N Y Acad Sci, 1124 (2008), pp. 1-38 
Buzsaki et al., 2007. G. Buzsaki, K. Kalia, M. Raichle. Inhibition and brain work. Neuron, 56 (2007), pp. 771-783

Cudeiro et al., 1997. J. Cudeiro, C. Rivadulla, R. Rodríguez, K.L. Grieve, S. Martínez-Conde, C. Acuña. Actions of compounds manipulating the nitric oxide system in the cat primary visual cortex. J Physiol (Lond), 504 (1997), pp. 467-478

Cudeiro et al., 2000. J. Cudeiro, C. Rivadulla, K.L. Grieve. A possible role for nitric oxide at the sleep/wake interface. Sleep, 23 (2000), pp. 829-835

de Labra et al., 2009. C. de Labra, C. Rivadulla, N. Espinosa, M. Dasilva, R. Cao, J. Cudeiro. Different sources of nitric oxide mediate neurovascular coupling in the lateral geniculate nucleus of the cat. Front Syst Neurosci, 3 (2009), p. 9

Duewell et al., 1996. S. Duewell, C.E. Kasserra, P. Jezzard, R.S. Balaban. Evaluation of methemoglobin as an autologous intravascular MRI contrast agent. Magn Reson Med, 35 (1996), pp. 787-789

Feelisch et al., 1996. M. Feelisch, D. Kubitzek, J. Werringloer. The oxyhemoglobin assay. M. Feelisch, J.S. Stamler (Eds.), Methods in nitric oxide research, Wiley, Chichester (1996), pp. 455-478

Francesconi et al., 1988. W. Francesconi, C.M. Müller, W. Singer. Cholinergic mechanisms in the reticular control of transmission in the cat lateral geniculate nucleus. J Neurophysiol, 59 (1988), pp. 1690-1718

Garthwaite, 1991. J. Garthwaite. Glutamate, nitric oxide and cell-cell signalling in the nervous system. Trends Neurosci, 14 (2) (1991), pp. 60-67

González-Mora et al., 2002. J.L. González-Mora, F.A. Martín, D. Rojas-Díaz, S. Hernández, I. RamosPérez, V.D. Rodríguez, M.A. Castellano. In vivo spectroscopy: a novel approach for simultaneously estimating nitric oxide and hemodynamic parameters in the rat brain. J Neurosci Methods, 119 (2002), pp. 151-161

Gujar et al., 2005. S.K. Gujar, S. Maheshwari, I. Björkman-Burtscher, P.C. Sundgren. Magnetic resonance spectroscopy. J Neuroophthalmol, 25 (3) (2005), pp. 217-226

Hartveit et al., 1993. E. Hartveit, S.I. Ramberg, P. Heggelund. Brain stem modulation of spatial receptive field properties of single cells in the dorsal lateral geniculate nucleus of the cat. J Neurophysiol, 70 (1993), pp. 1644-1655

Jones, 2003. B.E. Jones. Arousal systems. Front Biosci, 8 (2003), pp. s438-s451

Jones, 2004. B.E. Jones. Activity, modulation and role of basal forebrain cholinergic neurons innervating the cerebral cortex. Prog Brain Res, 145 (2004), pp. 157-169

Kelm et al., 1997. M. Kelm, R. Dahmann, D. Wink, M. Feelisch. The nitric oxide/superoxide assay. Insights into the biological chemistry of the NO/O-2 interaction. J Biol Chem, 272 (1997), pp. 99229932

Leung and Moody, 2010. G. Leung, A.R. Moody. MR imaging depicts oxidative stress induced by methemoglobin. Radiology, 257 (2010), pp. 470-476

Li et al., 1999. B. Li, K. Funke, F. Wörgötter, U.T. Eysel. Correlated variations in EEG pattern and visual responsiveness of cat lateral geniculate relay cells. J Physiol (Lond), 514 (1999), pp. 857-874

Malonek and Grinvald, 1996. D. Malonek, A. Grinvald. Interactions between electrical activity and cortical microcirculation revealed by imaging spectroscopy: implications for functional brain mapping. Science, 272 (1996), pp. 551-554

Mantini et al., 2011. D. Mantini, A. Gerits, K. Nelissen, J.-B. Durand, O. Joly, L. Simone, H. Sawamura, C. Wardak, G.A. Orban, R.L. Buckner, W. Vanduffel. Default mode of brain function in monkeys. J Neurosci, 31 (2011), pp. 12954-12962

Mariño and Cudeiro, 2003. J. Mariño, J. Cudeiro. Nitric oxide-mediated cortical activation: a diffuse wake-up system. J Neurosci, 23 (2003), pp. 4299-4307

Metherate et al., 1992. R. Metherate, C.L. Cox, J.H. Ashe. Cellular bases of neocortical activation: modulation of neural oscillations by the nucleus basalis and endogenous Ach. J Neurosci, 12 (1992), pp. $4701-4711$

McCormick and Bal, 1997. D.A. McCormick, T. Bal. Sleep and arousal: thalamocortical mechanisms. Annu Rev Neurosci, 20 (1997), pp. 185-215

Moncada et al., 1991. S. Moncada, R.M. Palmer, E.A. Higgs. Nitric oxide: physiology, pathophysiology, and pharmacology. Pharmacol Rev, 43 (2) (1991), pp. 109-142

Montague et al., 1991. P.R. Montague, C.D. Gancayco, M.J. Winn, R.B. Marchase, M.J. Friedlander. Role of NO production in NMDA receptor-mediated neurotransmitter release in cerebral cortex. Science, 263 (5149) (1991), pp. 973-977

Moruzzi and Magoun, 1949. G. Moruzzi, H.W. Magoun. Brain stem reticular formation and activation of the EEG. Electroencephalogr Clin Neurophysiol, 1 (1949), pp. 455-473 
Nitz et al., 2010. W.R. Nitz, T. Balzer, D.S. Grosu, T. Allkemper. Principles of magnetic resonance. P. Reimer, P.M. Parizel, J.F.M. Meanev, F.-A. Stichnoth (Eds.), Clinical MR imaging: a practical approach, Springer (2010)

Ogawa et al., 1990. S. Ogawa, T.M. Lee, A.S. Nayak, P. Glynn. Oxygenation-sensitive contrast in magnetic resonance image of rodent brain at high magnetic fields. Magn Reson Med, 14 (1990), pp. $68-78$

Ohgami et al., 2010. Y. Ohgami, E. Chung, R.M. Quock. Nitrous oxide-induced NO-dependent neuronal release of $\beta$-endorphin from the rat arcuate nucleus and periaqueductal gray. Brain Res, 1366 (2010), pp. 38-43

Pang et al., 2010. K.K. Pang, Y.S. Tsai, H.C. Chang, K.N. Hsu. Methemoglobin suppression in a 0.3 Tesla magnet: an in vitro and in vivo study. Acad Radiol, 17 (5) (2010), pp. 624-627

Pawela et al., 2008. C.P. Pawela, B.B. Biswal, Y.R. Cho, D.S. Kao, R. Li, S.R. Jones, M.L. Schulte, H.S. Matloub, A.G. Hudetz, J.S. Hyde. Resting-state functional connectivity of the rat brain. Magn Reson Med, 59 (2008), pp. 1021-1029

Rivadulla et al., 2011. C. Rivadulla, C. de Labra, K.L. Grieve, J. Cudeiro. Vasomotion and neurovascular coupling in the visual thalamus in vivo. PLoS One, 6 (2011), p. e28746

Schuman and Madison, 1994. E.M. Schuman, D.V. Madison. Nitric oxide and synaptic function. Annu Rev Neurosci, 17 (1994), pp. 153-183

Semba, 2000. K. Semba. Multiple output pathways of the basal forebrain: organization, chemical heterogeneity, and roles in vigilance. Behav Brain Res, 115 (2000), pp. 117-141

Sherin et al., 1996. J.E. Sherin, P.J. Shiromani, R.W. McCarley, C.B. Saper. Activation of ventrolateral preoptic neurons during sleep. Science, 271 (1996), pp. 216-219

Snyder and Bredt, 1991. S.H. Snyder, D.S. Bredt. Nitric oxide as a neuronal messenger. Trends Pharmacol Sci, 4 (1991), pp. 125-128

Steriade et al., 1988. M. Steriade, D. Paré, A. Parent, Y. Smith. Projections of cholinergic and noncholinergic neurons of the brainstem core to relay and associational thalamic nuclei in the cat and macaque monkey. Neuroscience, 25 (1988), pp. 47-67

Steriade et al., 1991a. M. Steriade, R.C. Dossi, A. Nuñez. Network modulation of a slow intrinsic oscillation of cat thalamocortical neurons implicated in sleep delta waves: cortically induced synchronization and brainstem cholinergic suppression. J Neurosci, 11 (1991), pp. 3200-3217

Steriade et al., 1991b. M. Steriade, R.C. Dossi, D. Paré, G. Oakson. Fast oscillations (20-40 Hz) in thalamocortical systems and their potentiation by mesopontine cholinergic nuclei in the cat. Proc Natl Acad Sci U S A, 88 (1991), pp. 4396-4400

Steriade et al., 1993a. M. Steriade, F. Amzica, A. Nuñez. Cholinergic and noradrenergic modulation of the slow (approximately $0.3 \mathrm{~Hz}$ ) oscillation in neocortical cells. J Neurophysiol, 70 (1993), pp. 13851400

Steriade et al., 1993b. M. Steriade, D.A. McCormick, T.J. Sejnowski. Thalamocortical oscillations in the sleeping and aroused brain. Science, 262 (1993), pp. 679-685

Steriade et al., 1997. M. Steriade, E.G. Jones, D.A. McCormick. Thalamus. Organization and function, vol. 1Elsevier, Oxford (1997), pp. 269-337

Swanson et al., 1987. L.W. Swanson, G.J. Mogenson, R.B. Simerly, M. Wu. Anatomical and electrophysiological evidence for a projection from the medial preoptic area to the 'mesencephalic and subthalamic locomotor regions' in the rat. Brain Res, 405 (1987), pp. 108-122

Szymusiak and McGinty, 1986. R. Szymusiak, D. McGinty. Sleep-related neuronal discharge in the basal forebrain of cats. Brain Res, 370 (1986), pp. 82-92

Szymusiak et al., 2001. R. Szymusiak, T. Steininger, N. Alam, D. McGinty. Preoptic area sleepregulating mechanisms. Arch Ital Biol, 139 (2001), pp. 77-92

Uhlrich et al., 1995. D.J. Uhlrich, N. Tamamaki, P.C. Murphy, S.M. Sherman. Effects of brain stem parabrachial activation on receptive field properties of cells in the cat's lateral geniculate nucleus. J Neurophysiol, 73 (1995), pp. 2428-2447

Wolfe and Palmer, 1998. J. Wolfe, L.A. Palmer. Temporal diversity in the lateral geniculate nucleus of cat. Vis Neurosci, 15 (1998), pp. 653-675

White et al., 2011. B.R. White, A.Q. Bauer, A.Z. Snyder, B.L. Schlaggar, J.-M. Lee, J.P. Culver. Imaging of functional connectivity in the mouse brain. PLoS One, 6 (2011), p. e16322

Zelinski et al., 2009. L.M. Zelinski, Y. Ohgami, R.M. Quock. Exposure to nitrous oxide stimulates a nitric oxide-dependent neuronal release of beta-endorphin in ventricular-cisternally-perfused rats. Brain Res, 1300 (2009), pp. 37-40

Zhang and Snyder, 1995. J. Zhang, S.H. Snyder. Nitric oxide in the nervous system. Annu Rev Pharmacol Toxicol, 35 (1995), pp. 213-233 
Zijlstra et al., 2000. W.G. Zijlstra, A. Buursma, O.W. Assendelft. Visible and near-infrared absorption spectra of human and animal hemoglobin: determination and application. VSP, Zeist, Netherlands (2000) 